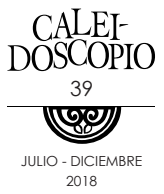

2018

\section{Percepción de autoeficacia y rendimiento académico en estudiantes de bachillerato}

Perception of self-efficacy and academic performance in high school students

\title{
RESUMEN
}

El objetivo de este artículo es abordar la relación entre la percepción de autoeficacia y el rendimiento académico en estudiantes de bachillerato. Participaron 592 jóvenes adscritos a un bachillerato de una universidad pública, respondiendo una ficha de identificación de datos personales, familiares y escolares, así como una Escala de Autoeficacia en Conductas Académicas (EACA). Además, se recuperó su promedio escolar global en el sistema escolar; se encontró una correlación estadísticamente significativa entre autoeficacia y el rendimiento académico $(r=0.409, p=.0000001)$. Para finalizar, se discute una posible relación de influencia recíproca y positiva entre la percepción de autoeficacia y el desempeño académico.

Palabras clave: rendimiento académico, autoeficacia, adolescentes.

\footnotetext{
1 Universidad de Guadalajara, México.

2 Universidad Michoacana de San Nicolás de Hidalgo, México.

3 Universidad de Colima, México.

4 Escuela Normal Superior de Especialidades, México.
} 
The aim of this paper is to address the relationship between the perception of self-efficacy and academic performance in students of high school. 592 teenagers from a public high school answered an identification card with questions regarding personal, familiar and scholar information, as well as a Scale of Self-Efficacy in Academic Behavior (EACA, in Spanish). Global school averages of participants were also obtained. A statistically significant correlation between self-efficacy and academic performance $(r=0.409, p=.0000001)$ was found. As a conclusion, a reciprocal, positive influence between perception of self-efficacy and academic performance is discussed.

Keywords: academic performance, self-efficacy, teenagers.

Bandura (1986) afirma que existen procesos cognitivos de autorregulación personal; uno de ellos es lo que denomina percepción de autoeficacia, que remite a los juicios personales y creencias sobre nuestras propias capacidades relacionadas con experiencias de dominio de situaciones. Esta percepción genera expectativas que se traducen en la convicción de que se puede ejecutar con éxito una conducta para producir determinados resultados, lo que sin duda influye en la elección y mantenimiento de determinados comportamientos.

La percepción de autoeficacia constituye un factor determinante que explica la elección de actividades, cuánto esfuerzo se invertirá en la realización de una tarea y cuánto tiempo se va a mantener ese esfuerzo al enfrentar situaciones estresantes. En este sentido, el logro de resultados esperados depende, en buena parte, de lo que una persona considera que va a ser capaz de hacer en determinadas situaciones. Bandura (1986) afirma que los resultados que una persona espera derivan en su mayor parte de los propios juicios, de tal forma que el éxito eleva las expectativas de dominio, mientras que el fracaso las disminuye.

El éxito repetido reduce la influencia del fracaso, mientras que los fracasos ocasionales anulados por esfuerzos posteriores pueden reforzar la persistencia automotivada, al darse cuenta la persona, por experiencia, de que incluso los obstáculos más difíciles pueden superarse con un esfuerzo continuado. Dominar tareas que nos superan es un reto que aumenta la propia competencia. Las personas que disponen de un nivel de autoeficacia elevado visualizan los escenarios de ma- 
nera positiva, promueven el desarrollo de actividades e incrementan sus conductas a dominar y, como consecuencia, amplían su número de competencias desarrolladas y se mantienen orientadas a las tareas usando generalmente un buen pensamiento analítico.

Bandura (1995) afirmó que el concepto de autoeficacia académica no hace referencia a las habilidades de las que dispone el sujeto que le permiten dominar las circunstancias continuamente cambiantes del entorno académico en el que se desenvuelve, sino a la opinión que éste tiene sobre lo que puede o no hacer con ellas. Alegre (2014) menciona que el proceso de autoeficacia constituye la evaluación idiosincrática que media entre la situación o tarea, la activación del estudiante y su desempeño; es decir, a mayor nivel de expectativas de la eficacia académica personal, mayor será el esfuerzo desplegado y el tiempo dedicado para alcanzar las metas de aprendizaje por parte del estudiante.

Por su parte, Colom (2012) considera que si un estudiante tiene habilidades óptimas y existen adecuados incentivos, las expectativas sobre la propia eficacia se convierten en un determinante esencial del tipo de actividad que elegirá, cuánto esfuerzo invertirá en ella y durante cuánto tiempo se esforzará por gestionar las conductas que produzcan estrés. Los alumnos con un elevado sentido de eficacia para cumplir tareas educativas persistirán más ante dificultades, trabajarán con más intensidad y participarán más que aquellos que duden de sus capacidades.

De acuerdo a la teoría, la autoeficacia contribuye al rendimiento académico, el cual es el resultado del proceso de enseñanza-aprendizaje (Tournon, 1984). El rendimiento es calculado a través de una evaluación en la que se indica en qué medida el proceso educativo logra sus objetivos fundamentales y confronta los fijados con los realmente alcanzados (Cruz y Quiñones, 2012). Generalmente, esta evaluación se representa mediante una estimación numérica a partir de criterios de logro previamente establecidos que refleja el nivel demostrado de conocimientos, por ello, consideramos que el rendimiento académico puede evidenciarse a partir de la calificación.

En los últimos años se han llevado a cabo algunas investigaciones respecto a la percepción de autoeficacia y su relación con algunas variables académicas, tales como estrategias de afrontamiento (Domínguez, 2016), práctica física y deportiva (Oropeza, Ávalos y Ferreyra, 2017; Kyle, Hernández, Reigal y Morales, 2016), autoesti-

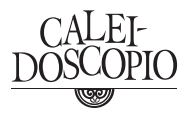


ma positiva (Faurie, 2015), madurez vocacional (Santana, Feliciano y Santana, 2013) y persistencia académica (Wright, Jenkins y Murdock, 2012), encontrando una relación positiva.

Referente al rendimiento académico y su relación con la autoeficacia, diversos estudios confirman la dependencia entre ellas (Castellanos, Latorre, Mateus y Navarro, 2017; Feldman y Kubota, 2015; Hoigaard, Kova, Overby y Haugen, 2015; Parker, Marsh, Ciarrochi, Marshal y Abduljabbar, 2014; DiBenedetto y Bembenutti, 2013; Barca, Almeida, Porto, Peralbo y Brenlla, 2012; Ornelas, Blanco, Gastélum y Chávez, 2012; Rosario et al., 2012). En su mayoría, tales estudios se realizaron en otros países y con población universitaria. Por ello, nuestro objetivo de investigación fue relacionar y comparar la percepción de autoeficacia y el rendimiento académico en estudiantes de bachillerato de una escuela pública de México.

\section{MÉTODO}

\section{Participantes}

La muestra fue determinada mediante un procedimiento no probabilístico, seleccionando a los participantes por conveniencia. Colaboraron voluntariamente 592 estudiantes regulares que, al momento de la recolección de la información, cursaban primero (22\%), tercero $(29 \%)$ o quinto (49\%) semestre de bachillerato; $46 \%$ eran hombres y $54 \%$ mujeres. Se consideraron cuatro bachilleratos generales ubicados en una zona urbana. En cuanto a criterios de inclusión, se tomaron en cuenta a los estudiantes que desearon participar voluntariamente y estuvieron en el momento de la aplicación de las encuestas, respondiendo por completo todos los formatos de evaluación y firmando la carta de consentimiento informado.

\section{Instrumentos}

Utilizamos una ficha de identificación que indagó mediante preguntas cerradas de opción múltiple lo referente a datos personales (edad, sexo, estado civil, etc.), familiares (nivel de estudios de los padres, expectativas, etc.) y académicos (semestre cursado, promedio escolar, 
etc.). Para esta última información se pidió a los estudiantes que consultaran al sistema escolar.

Para evaluar la percepción de autoeficacia, utilizamos la Escala de Autoeficacia en Conductas Académicas (EACA), diseñada por Blanco, Ornelas, Viciana y Rodríguez (2016). Es una encuesta de 13 reactivos tipo Likert relacionados con conductas académicas. El encuestado indica la frecuencia con la que actualmente realizaría o manifestaría una conducta, donde 0 corresponde a la frecuencia nula de la conducta y 5 a la frecuencia ideal. El instrumento considera tres dimensiones (Borzone, 2017): 1) atención (la focalización sobre las actividades académicas); 2) comunicación (intercambio y difusión de información con fines académicos); y 3) excelencia (adherencia a las normas presentes en el contexto académico). En cuanto a consistencia interna, los valores Alfa de Cronbach en las dimensiones de esta escala son superiores a 0.70 (Blanco, Martínez, Zueck y Gastélum, 2011).

\section{Procedimiento}

El diseño del estudio fue cuantitativo, transversal, descriptivo y analítico. Previamente, las autoridades de las escuelas firmaron la carta de consentimiento informado con el fin de cuidar los criterios éticos de la investigación. La recolección de la información por parte de los estudiantes se llevó a cabo en las aulas habituales en las que tomaban sus clases. El procesamiento y análisis de los datos se realizó con el paquete estadístico SPSS versión 21.0. Se aplicó estadística descriptiva: medias, desviación típica y frecuencias simples. Se estimaron medidas de correlación de Sperman y Pearson entre las variables relevantes, así como medidas de diferencias mediante la prueba $\mathrm{T}$ para grupos independientes, con el objetivo de comparar datos personales y familiares. Se tomó como criterio de significancia estadística un valor $p$ menor o igual a 0.05 .

\section{RESULTADOS}

Participaron 592 estudiantes de bachillerato, con una edad promedio de 16.5 años, en un rango de 14 a 21 años ( $D E=1.03$ ). 20\% acudían a la escuela en el turno matutino, $34 \%$ en el turno vespertino y $45 \%$ en el turno nocturno. Predominantemente eran solteros (86\%) y sólo $34 \%$

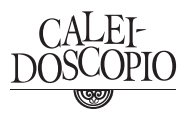


laboraban además de estudiar. La aplicación se llevó a cabo en cuatro escuelas de educación media superior de una ciudad en México: Bachillerato \#30 (20\%), Bachillerato \#15 (16\%), Bachillerato \#12 (19\%) y Bachillerato \#3 (45\%).

Respecto al rendimiento académico, el cual fue definido a partir del promedio escolar global cuantificado en una escala de 0 a 10 , los participantes mostraron una calificación promedio de $8.57(\mathrm{DE}=0.61$ ). Con una prueba $\mathrm{T}$ para grupos independientes, comparamos el promedio escolar y el sexo, encontrando una diferencia estadísticamente significativa $(t=-2.654, g l=588, p=.04)$; las mujeres presentaron un promedio de calificación más alto (8.6).

En la Tabla 1 se observa el promedio escolar de acuerdo con el bachillerato de adscripción. Mediante la prueba ANOva de un factor no encontramos diferencias estadísticamente significativas $(\mathrm{F}=2.261, \mathrm{gl}=$ $3, p=.084)$ entre el bachillerato de adscripción de los participantes y el promedio general de calificaciones. Al indagar acerca del semestre cursado y el promedio general de calificaciones, observamos algunas diferencias estadísticamente significativas (ANOVA de un factor), entre ellas $\mathrm{F}(2)=16.750(p=.0000001)$. Para identificar entre qué semestres había estas diferencias, realizamos la prueba post hoc HSD de Tukey y encontramos que ocurrieron entre el primer y el tercer semestre ( $p=$ $.000014)$, y entre el primer y el quinto semestre $(p=.0000001)$.

Tabla 1. Resultados descriptivos del promedio por bachillerato y global

\begin{tabular}{lcccc}
\hline \multicolumn{1}{c}{ Bachillerato } & MIN & MÁX & M & DE \\
\hline$\# 3$ & 7.00 & 9.95 & 8.52 & 0.58 \\
$\# 12$ & 6.00 & 9.80 & 8.58 & 0.71 \\
$\# 15$ & 6.00 & 9.80 & 8.54 & 0.64 \\
$\# 30$ & 7.00 & 9.80 & 8.70 & 0.52 \\
\hline General & 6.00 & 9.95 & 8.57 & 0.61 \\
\hline
\end{tabular}

$n=592$

Respecto a la autoeficacia podemos apreciar que, en su mayoría, los participantes obtuvieron un nivel alto tanto en la percepción de 
autoeficacia global como en las dimensiones de atención y excelencia. La proporción fue menor en la dimensión comunicación, especialmente en el caso de las mujeres (Tabla 2). Mediante una prueba T para grupos independientes, comparamos el puntaje total de autoeficacia y el sexo. A pesar de que las mujeres obtuvieron un puntaje ligeramente mayor que los hombres, no hubo diferencias estadísticamente significativas entre ambos grupos.

Tabla 2. Distribución porcentual de la percepción de autoeficacia global y sus dimensiones de acuerdo al sexo

\begin{tabular}{ccccccc}
\hline & \multicolumn{5}{c}{ Sexo y nivel de autoeficacia } \\
\cline { 2 - 7 } Dimensiones & \multicolumn{3}{c}{ Hombres } & \multicolumn{4}{c}{ Mujeres } \\
\cline { 2 - 7 } & Bajo & Medio & Alto & Bajo & Medio & Alto \\
\hline Atención & 0.4 & 16.4 & 83.2 & 0.9 & 12.6 & 86.5 \\
Excelencia & 0.7 & 15.7 & 83.6 & 0.9 & 11.0 & 88.1 \\
Comunicación & 3.3 & 27.4 & 69.3 & 9.1 & 30.2 & 60.7 \\
\hline Global & 0.0 & 16.8 & 83.2 & 0.6 & 16.7 & 82.7 \\
\hline
\end{tabular}

$n=592$

Al calcular el promedio escolar en función de los niveles obtenidos en la escala de autoeficacia y sus dimensiones, constatamos que a mayor puntaje de autoeficacia el promedio escolar es mayor (Tabla 3). Cuando comparamos por hombres y mujeres los niveles de autoeficacia y el promedio escolar, también observamos la misma relación entre ambas variables. 
Tabla 3. Promedio escolar de acuerdo a los puntajes de autoeficacia

\begin{tabular}{cccccc}
\hline Dimensión & Nivel autoeficacia & MIN & MÁX & M & DE \\
\hline Atención & Bajo & 7.6 & 9.2 & 8.4 & 0.7 \\
& Medio & 6.0 & 9.8 & 8.2 & 0.7 \\
Excelencia & Alto & 6.0 & 10.0 & 8.6 & 0.6 \\
& Bajo & 6.5 & 8.8 & 7.7 & 0.9 \\
& Medio & 6.0 & 9.2 & 8.0 & 0.6 \\
Comunicación & Alto & 6.0 & 10.0 & 8.7 & 0.6 \\
& Bajo & 7.0 & 9.6 & 8.3 & 0.6 \\
& Medio & 6.0 & 9.9 & 8.4 & 0.6 \\
& Alto & 6.0 & 10.0 & 8.7 & 0.6 \\
\hline \multirow{2}{*}{ Global } & Bajo & 7.0 & 7.6 & 7.3 & 0.4 \\
& Medio & 6.0 & 9.7 & 8.2 & 0.6 \\
& Alto & 6.0 & 10.0 & 8.7 & 0.6 \\
\hline
\end{tabular}

$n=592$

Aunado a esto, se calculó el coeficiente de correlación de Pearson entre el puntaje global de autoeficacia y el promedio escolar actual. El resultado arroja una correlación moderada estadísticamente significativa $(r=0.409, p=.0000001)$ entre ambas variables. De manera más específica, comparamos cada una de las dimensiones de la escala de autoeficacia por sexo, observando diferencias estadísticamente significativas en todas las dimensiones, pero no en el puntaje global (Tabla 4). 
Tabla 4. Diferencias por sexo entre los puntajes de percepción de autoeficacia

\begin{tabular}{|c|c|c|}
\hline Dimensión & Sexo & Pruebat \\
\hline Atención & $\begin{array}{l}\text { Mujeres: } M=42.10 \\
\text { Hombres: } M=41.05\end{array}$ & $t(590)=-1.913, p=.023$ \\
\hline Excelencia & $\begin{array}{l}\text { Mujeres: } M=34.5 \\
\text { Hombres: } M=33.2\end{array}$ & $t(590)=-2.912, p=.002$ \\
\hline Comunicación & $\begin{array}{l}\text { Mujeres: } M=34.5 \\
\text { Hombres: } M=30.4\end{array}$ & $t(590)=2.309, p=.011$ \\
\hline Global & $\begin{array}{l}\text { Mujeres: } M=105.7 \\
\text { Hombres: } M=104.7\end{array}$ & $t(590)=-0.759, p=.224$ \\
\hline
\end{tabular}

$n=592$

Finalmente, realizamos un ANOVA de un factor entre el bachillerato de adscripción y el puntaje global de autoeficacia. No se encontraron diferencias estadísticamente significativas $(\mathrm{F}=2.081, g l=3, p=.102)$. Respecto a las desigualdades entre las dimensiones de autoeficacia y el bachillerato de adscripción, únicamente encontramos diferencias con la dimensión comunicación ( $\mathrm{F}=4.167, \mathrm{gl}=3, p=.006)$. Para observar entre qué bachilleratos había diferencias estadísticamente significativas, se hizo la prueba post hoc HSD de Tukey y se encontró que fueron entre el Bachillerato \#30 y el Bachillerato \#15 ( $p=.016)$, y entre el Bachillerato \#30 y el Bachillerato \#12 ( $p=.015)$. No encontramos desigualdades significativas entre el puntaje global de autoeficacia y el semestre cursado, ni entre el rendimiento académico y el semestre cursado.

\section{DISCUSIÓN}

Nuestros hallazgos confirman los reportados por otras investigaciones respecto a la relación estrecha y positiva que tienen la percepción de autoeficacia y el rendimiento académico (Feldman y Kubota, 2015; Hoigaard, Kova, Overby y Haugen, 2015; Parker, Marsh, Ciarrochi, Marshal y Abduljabbar, 2014; DiBenedetto y Bembenutti, 2013; Barca, Almeida, Porto, Peralbo y Brenlla, 2012; Ornelas, Blanco, Gastélum y 
Chávez, 2012; Rosario et al., 2012). La dimensión comunicación fue la que puntuó más bajo; ello coincide con lo encontrado por Ornelas y cols. (2012), quienes interpretaron el hallazgo como área de oportunidad para mejorar los niveles de autoeficacia.

Zeldin (2000) señala que las creencias de eficacia ocupan un rol mediacional en el funcionamiento humano, actuando a modo de filtro entre las habilidades y logros del ser humano, lo cual podría explicar los niveles altos en cuanto a excelencia y atención, que sobresalen en esta variable. Por su parte, Edel (2003) afirma que percibirse como hábil (capaz) es un elemento central, considera que el juego de valores habilidad-esfuerzo se torna riesgoso para los alumnos y puede determinar el sentimiento de orgullo y satisfacción sobre sí mismo.

Las mujeres puntuaron más alto en las dimensiones de excelencia y atención, diferencia mínima aunque estadísticamente significativa. Esto podría explicarse mediante la definición propuesta por Borzone (2017), en el sentido de que las mujeres presentan mayor capacidad para hacer frente a situaciones específicas. Ello involucra la creencia acerca de las propias capacidades para organizar y ejecutar acciones para alcanzar determinados resultados. Esta creencia se relaciona también con niveles altos en las dimensiones de excelencia y atención, es decir, se atribuyen una mayor focalización sobre las actividades académicas y adherencia a las normas presentes en el contexto académico.

Por otra parte, resulta interesante que los hombres puntúen ligeramente más alto en comunicación, es decir, que presenten mayor autopercepción en el uso de habilidades y capacidades cognitivas para el intercambio y difusión de información con fines académicos. De manera importante, observamos la relación que guardan nuestras variables de estudio, ya que al compararlas encontramos diferencias significativas entre ellas, de tal forma que podemos suponer que los estudiantes con una adecuada percepción de autoeficacia tenderán a evidenciar un alto nivel de desempeño académico y viceversa. En nuestra investigación también resaltan variables intervinientes que en menor medida afectan esta situación, como ocurre con el sexo y las características de la escuela. La presente investigación contribuye a la explicación de estos procesos subjetivos involucrados en los contextos escolares, los cuales, tal como se mencionó en líneas anteriores, son útiles para comprender al estudiantado y poder ofrecer herramientas que garanticen su permanencia y la culminación de sus estudios. 
También observamos relaciones fuertes entre el rendimiento académico (promedio global de calificaciones), el sexo, el tipo de bachillerato y el semestre, lo cual constata la necesidad de abordar y comprender el tema desde un enfoque multidimensional, en el que, además de la variable psicológica aquí abordada, se considere la interrelación entre distintos factores sociales y escolares que lo afectan.

Finalmente, es importante señalar la relevancia de continuar este tipo de investigaciones en otros contextos al considerar la indagación de otras variables que pudieran estar involucradas. Del mismo modo, resultaría conveniente complementar este tipo de investigaciones cuantitativas con aproximaciones cualitativas que nos permitan entender estas variables psicológicas, desde la propia subjetividad de los participantes.

\section{REFERENCIAS}

Alegre, A. (2014). Autoeficacia académica, autorregulación del aprendizaje y rendimiento académico en estudiantes universitarios iniciales. Propósito y Representaciones, 2(1), 79-120. DOI: 10.20511/pyr2014. v2n 1.54 .

Bandura, A. (1986). The explanatory and predictive scope of self-efficacy theory. Journal of Clinical and Social Psychology, 4, 359-373.

Bandura, A. (1995). Self-efficacy in changing societies. Cambridge: Cambridge University Press.

Barca, A., Almeida, L., Porto, A., Peralbo, M. y Brenlla, J. (2012). Motivación escolar y rendimiento: impacto de metas académicas, de estrategias de aprendizaje y autoeficacia. Anales de Psicología, 28(3), 848-859. DOI: 10.6018/analesps.28.3.156221.

Blanco, J., Ornelas, M, Viciana, J. y Rodríguez, J. (2016). Composición factorial de una escala de autoeficacia en el cuidado de la alimentación y salud física en universitarios mexicanos. Nutrición Hospitalaria, 33(2), 379-385. DOI: 10.20960/nh. 120.

Blanco, H., Martínez, M., Zueck, M. C. y Gastélum, G. (2011). Análisis psicométrico de la escala Autoeficacia en Conductas Académicas en unviersitarios de primer ingreso. Revista Electrónica Actualidades Investigativas en Educación, 11(3), 1-27. Recuperado de http://www.redalyc.org/pdf/447/44722178003.pdf.

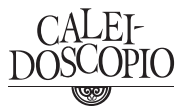


Borzone, M. (2017). Autoeficacia y vivencias académicas en estudiantes universitarios. Acta Colombiana de Psicología, 20(1), 266-274.

Castellanos, V., Latorre, D., Mateus, S. y Navarro, C. (2017). Modelo explicativo del desempeño académico desde la autoeficacia y los problemas de conducta. Revista Colombiana de Psicología, 26(1), 149-161. Recuperado de: http://www.redalyc.org/articulo.oa?id=80454273010.

Colom, R. (2012). Psicología de las diferencias individuales: Teoría y práctica. Madrid: Pirámide.

Cruz, N. y Quiñones, A. (2012). Importancia de la evaluación y autoevaluación en el rendimiento académico. Revista Zona Próxima, 16. Recuperado de: http://rcientificas.uninorte.edu.co/index.php/zona/ article/download/3062/2760.

DiBenedetto, M. y Bembenutti, H. (2013). Within the pipeline: Selfregulated learning, self-efficacy, and socialization among college students in science courses. Learning and Individual Differences, 23, 218-224. DOI: 10.1016/j.lindif.2012.09.015.

Domínguez, S. (2016). Afrontamiento ante la ansiedad pre-examen y autoeficacia académica en estudiantes de ciencias de la salud. Educación Médica, 99, 1-4. DOI:10.1016/j.edumed.2016.07.007.

Edel, R. (2003). El rendimiento académico: concepto, investigación y desarrollo. Revista Iberoamericana sobre Calidad, Eficacia y Cambio en Educación, 1(2). Recuperado de http://www.ice.deusto.es/rinace/reice/volln2/Edel.pdf.

Faurie, I. (2015). Transition lycée-université: relations entre indécision vocationnelle, sentiment d'efficacité personnelle et stress perçu. Colloque international: Apprendre, Transmettre, Innover à et par l'Université. Junio 2015, Montpellier (France). DOI: 10.21409/HAL01278363.

Feldman, D. y Kubota, M. (2015). Hope, self-efficacy, optimism, and academic achievement: Distinguishing constructs and levels of specificity in predicting college grade-point average. Learning and Individual Differences, 37, 210-216. DOI: 10.1016/j.lindif.2014.11.022.

Hoigaard, R., Kova, V. B., Overby, N. C. y Haugen, T. (2015). Academic self-efficacy mediates the effects of school psychological climate on academic achievement. School Psychology Quarterly, 30(1), 64-74. DOI: $10.1037 / \mathrm{spq} 0000056$.

Kyle, T.L., Hernández, A., Reigal, R. y Morales, V. (2016). Efectos de la actividad física en el autoconcepto y la autoeficacia en preadoles- 
centes. Retos, 29, 61-65. Recuperado de https://recyt.fecyt.es/index. php/retos/article/view/36873.

Ornelas, M., Blanco, H., Gastélum, G. y Chávez, A. (2012). Autoeficacia percibida en la conducta académica de estudiantes universitarias. Formación Universitaria, 5(2), 17-22. DOI: 10.4067/S071850062012000200003.

Oropeza, R., Ávalos, M. y Ferreyra, D. (2017). Comparación entre rendimiento académico, autoeficacia y práctica deportiva en estudiantes universitarios. Revista Actualidades Investigativas en Educación, 17(1), 1-21. DOI: 10.15517/aie.v17i1.27271.

Parker, P., Marsh, H., Ciarrochi, J., Marshal, S. y Abduljabbar, A. (2014). Juxtaposing math self-efficacy and self-concept as predictors of long-term achievement outcomes. Educational Psychology, 34, 29-48. DOI: 10.1080/01443410.2013.797339.

Rosario, P., Lourenço, A., Paiva, M., Núñez, J., González, J. y Valle, A. (2012). Autoeficacia y utilidad percibida como condiciones necesarias para un aprendizaje académico autorregulado. Anales de Psicología, 28(1), 37-44. Recuperado de: http://revistas.um.es/analesps/article/view/140502.

Santana, L., Feliciano, L. y Santana, J. (2013). Madurez y autoeficacia vocacional en $3^{\circ}$ y $4^{\circ}$ de ESO, bachillerato y ciclos formativos. Revista Española de Orientación y Psicopedagogía, 24(3), 8-26. Recuperado de: https://www2.uned.es/reop/pdfs/2013/24-3\%20-\%20Santana.pdf.

Tournon, J. (1984). Factores del rendimiento académico en la universidad. España: Ediciones Universidad de Navarra.

Wright, S., Jenkins, A. y Murdock, J. (2012). Career Development Among First-Year College Students. Journal of Career Development, 40(4). 292310. DOI: 10.1177/0894845312455509.

Zeldin, A. L. (2000). Sources and Effects of the Self-Efficacy Beliefs of Men with Careers in Mathematics, Science, and Technology (tesis doctoral). Atlanta: Emory University. Recuperado de: https://www.uky.edu/ eushe2/ Pajares/ZeldinDissertation2000.PDF.

\section{AGRADECIMIENTOS}

Este proyecto se llevó a cabo gracias al financiamiento de PRODEP con el número de liberación DSA/103.5/16/10406. Agradecemos la colaboración de las pasantes en psicología Maritza Guadalupe Hernández

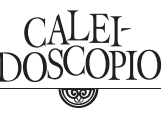


Aguilar, Andrea Susana Pérez García y Rita Berenice Miranda Álvarez. Así también a Nancy Patricia Valencia Silva, becaria del Programa de Estímulos Económicos a Estudiantes Sobresalientes de la Universidad de Guadalajara.

\section{ACERCA DE LOS AUTORES}

María Luisa Ávalos Latorre (luisa.avalos@academicos.udg.mx) es profesora e investigadora en el Centro Universitario de Tonalá de la Universidad de Guadalajara. Maestra en Ciencia del Comportamiento con Opción en Análisis de la Conducta y doctora en Psicología por la Universidad de Guadalajara (ORCID 0000-0002-1183-1518).

Roberto Oropeza Tena (scherzo2112@yahoo.com) es profesor e investigador en la Facultad de Psicología de la Universidad Michoacana de San Nicolás de Hidalgo. Maestro en Análisis Experimental de la Conducta y doctor en Psicología de la Salud por la Universidad Nacional Autónoma de México (ORCID 0000-0002-2561-2164).

José Carlos Ramírez Cruz (jose_ramirez29@ucol.mx) es docente en la Facultad de Psicología de la Universidad de Colima. Licenciado en Psicología y maestro en Educación por la Universidad Virtual del Estado de Guanajuato (ORCID 0000-0002-1224-4382).

María Úrsula Palos Toscano (ursulapalos@hotmail.com) es profesora e investigadora de la Escuela Normal Superior de Especialidades de la Secretaría de Educación Jalisco. Maestra en Psicología Clínica y doctora en Psicología por la Universidad de Guadalajara (ORCID 00000001-5024-8889).

\section{CÓMO CITAR ESTE ARTÍCULO}

Ávalos, M. L., Oropeza, R., Ramírez, J. C. y Palos, M. U. (2018). Percepción de autoeficacia y rendimiento académico en estudiantes de bachillerato. Caleidoscopio. Revista Semestral de Ciencias Sociales y Humanidades, 22(39). DOI: 10.33064/39crscsh 1323 